\title{
Global optimization of multistage binary separation networks
}

\author{
Christian Kunde ${ }^{\mathrm{a}, 1}$, Achim Kienle ${ }^{\mathrm{a}, \mathrm{b}}$ \\ ${ }^{a}$ Otto von Guericke University Magdeburg, Universitätsplatz 2, 39106 Magdeburg, Germany \\ ${ }^{b}$ Max Planck Institute for Dynamics of Complex Technical Systems, Sandtorstraße 1, 39106 \\ Magdeburg, Germany
}

\begin{abstract}
This work covers general multistage binary separations with application examples in cooling crystallization, evaporative crystallization and organic solvent nanofiltration. Deterministic global optimization is applied to identify optimal configurations of multistage separation networks and study their sensitivity to parameter values. Superstructure optimization is conducted for countercurrent cascades and also for general superstructures to identify new multistage configurations. Results show substantially reduced separation effort for alternative configurations in large parameter regions, specifically in regions where optimal countercurrent cascades have a low number of stages. General, simple design guidelines for multistage separation with a low number of stages are derived from rigorous global optimization by comparing results for different processes. Keywords: multistage separation, MINLP, deterministic global optimization, cooling crystallization, solution crystallization, nanofiltration
\end{abstract}

\section{Introduction}

Economical and ecological efficiency of chemical production processes are determined by not only the reaction step but also the separation or purification of products. Development and optimization of efficient separation processes

\footnotetext{
${ }^{1}$ Corresponding author: christian.kunde@ovgu.de
}

Preprint submitted to Chemical Engineering and Processing: Process IntensificationJune 26, 2018 
5 therefore remain important topics in process engineering.

Homogeneous mixtures can be separated into their components using masstransfer operations; transporting matter from one homogeneous phase to another using a difference in chemical potential as a driving force [1]. Desired components are concentrated in the product phase by utilizing differences in 10 e.g. volatility, solubility or diffusivity, while other components remain in the residual phase. A variety of unit operations for separation are available such as distillation, crystallization, extraction and filtration.

Yield and product purity of a single separation unit are often insufficient for a given separation task due to limited equilibrium conditions, relative transfer rates of different components or other effects. In that case, multiple separation units can be employed to meet the requirements. The standard approach to such multistage separation processes using multiple units of the same type are countercurrent cascades, where the product phase is transported in the opposite direction to the residual phase.

20 Additional degrees of freedom are gained when allowing different types of separation units for each stage or entirely different separation processes in a hybrid separation scheme. However, this is not considered in the present work.

Countercurrent cascades are deployed for a range of industrially relevant separation processes [1], including cooling crystallization cascades [2, 3, 4, 5], evapora-

25 tive crystallization cascades [6, 7, 8, 9], organic solvent nanofiltration cascades [10, 11, and reverse osmosis cascades [12, 13, 14, 15].

Countercurrent contacting of phases improves the separation efficiency by increasing the overall driving force for mass transfer. However, the ideal countercurrent case is not well approximated by cascades with a low number of discrete separation stages. Better multistage configurations can be found by relaxing the restriction of countercurrent cascades.

Such general configurations have been studied extensively for multistage gas permeation processes, e.g. for the separation of $\mathrm{CO}_{2}$ and $\mathrm{CH}_{4}$ [16]. It was found in [17. that a three-stage configuration with one stage in series with a two-stage 35 countercurrent cascade is economically better than a two-stage cascade alone. 
The sensitivity of the optimal choice from a set of ten configurations with up to three stages to parameter values was studied in [18]. In [19], a method for multi-objective optimization of general gas separation processes with regard to energy consumption and membrane area was presented and applied to analyze 40 the parameter sensitivity of different two-stage configurations. A superstructure for simultaneous optimization of structural and operating parameters of general gas permeation networks was proposed in [20] and used in later studies, e. g. for finding optimal configurations for $\mathrm{CO}_{2}$ capture from flue gas [21] and from natural gas [22]. A different superstructure was deployed in [23, 24] to identify the optimal configuration for a biogas upgrading process using deterministic global optimization.

In our previous work [25, 26], optimal multistage configurations for cooling crystallization of binary mixtures are studied and improved configurations for this type of process are identified.

50 Structural optimization of separation networks has also been studied extensively for the synthesis of mass exchanger networks (MENs), which was first introduced in [27. However, MENs utilize external mass separating agents (e. g. solvents for liquid-liquid extraction) to remove selected components from product streams, which is not considered in the present work. For an overview on methods for 55 the synthesis of MENs we refer to [28, 29] and the references therein. Separation networks are also commonly used for reverse osmosis and nanofiltration with applications e.g. in seawater desalination and wastewater treatment. For these processes, it is important to distinguish between networks in which only retentate is reprocessed in subsequent units and those with reprocessing of permeate [30]. The former type of network often combines modules connected in parallel and retentate-connected modules in so-called tapered structures. This strategy allows to increase the effective membrane area of a separation stage [30] while taking into account technical limitations of membrane modules such as maximum flow rate or pressure loss and minimizing negative effects such as concentration polarization [27, 31. The latter type of network enables a higher maximum product quality by letting the permeate pass through more than one 
membrane. Membrane separation networks of this type are referred to by different names such as permeate-staged [32], multi-pass [33, 34] or multistage [33] configurations. Note that each pass of multi-pass configurations is usually designed as a tapered structure. Single-pass configurations are a standard choice for membrane separation processes with moderate product quality demands, while multi-pass configurations are used to achieve higher product qualities that are infeasible with single-pass configurations. As an example, when using reverse osmosis for seawater desalination, multi-pass configurations are necessary to reduce boron levels to recommended values [35]. Additionally, product recovery can be increased for multi-pass configurations by introducing recycles between passes, typically resulting in countercurrent cascades. While it is not generally applied, there is a considerable number of publications concerning countercurrent reverse osmosis. Two-pass reverse osmosis for seawater desalination is discussed in [36] for different configurations including countercurrent cascades. In [34, the energy consumption of two-pass membrane desalination is compared to a single-pass design. If single-pass is infeasible, using recycles in a countercurrent cascade can reduce energy consumption. Although countercurrent cascades are not considered in [33], recycling of the permeate of the second stage in a countercurrent design would reduce the feed salinity in this two-pass reverse osmosis process for waste water treatment. Two-pass countercurrent reverse osmosis is used in seawater desalination plants e.g. in Taranto (Italy) [13] and Tobyhanna Army Deport (USA) [14]. Countercurrent reverse osmosis for landfill leacheate is also modeled and tested in a pilot plant in [15]. A three-pass reverse osmosis process is employed in a seawater desalination plant in Saudi Arabia [12], combining one pass of a high pressure membrane with a two-pass countercurrent configuration using a low pressure membrane. A modified twopass countercurrent design deploying different membrane types within single passes is used in a seawater desalination plant in Adelaide (Australia) [37. Us95 ing nanofiltration for seawater desalination in a two-pass countercurrent process was studied and field-tested in Long Beach (USA) [38. They considered using similar and different membranes for different stages as well as pH-adjustment. 
In this work, the focus for membrane networks lies on multi-pass separation utilizing one type of membrane.

The present work provides new insight into general multistage separation processes based on extensive parameter studies beyond classical countercurrent cascades. Three exemplary separation processes are characterized in a setting of general separation units to establish comparability between different types of processes. The considered task is the separation of a two-component feed mixture into products with defined purity constraints, using multiple units with the same separation characteristic. Optimal configurations for each process and their sensitivity to parameter values are determined as a data basis for discussing common properties and design guidelines for general separation processes. Main results thereof are new configurations with substantially reduced separation effort compared to countercurrent cascades commonly applied for the considered processes. The structural and operational optimization of multistage separation processes performed in this work requires solving mixed-integer nonlinear problems with possibly many suboptimal local solutions. Finding globally optimal solutions of such problems is ensured by using a branch-and-bound-type deterministic global solver. As such, the present work extends the methods and results presented in $[39$.

The remaining part of this paper is structured as follows. In Section 2 models for general multistage separation of two-component mixtures independent of underlying separation processes are introduced, including a superstructure 120 for separation networks. In Section 3, unit models for processes utilizing cooling crystallization, organic solvent nanofiltration and evaporative crystallization are described. Results of the optimization problem defined in Section 4 are presented and discussed in Section 5. A conclusion is given in Section 6 . 


\section{General models}

\section{2.1. General separation unit}

A general separation unit as proposed in [1] is used in the present work as a common framework for different separation processes. As depicted in Figure 1 a feed molar flow $F$ is separated into a primary product $V$ and a secondary product $L$, with the $V$ phase being the processed output of the separation unit, e. g. the solid phase for crystallization or the permeate for filtration, and the $L$ phase being the non-processed residual output, e.g. the remaining mother liquor for crystallization and the retentate for filtration. For binary mixtures,

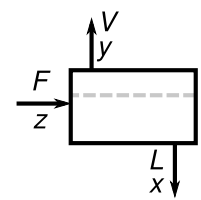

Figure 1: General separation unit.

the purpose of a separation unit is to concentrate a primary component $\mathrm{A}$ in the primary product and a secondary component $\mathrm{B}$ in the secondary product. The distribution of feed to primary product is defined in the present work by the unit yield $Y_{\mathrm{V}}$ as

$$
V=Y_{\mathrm{V}} F
$$

which also determines the secondary product due to mass conservation $F=$ $V+L$. The distribution coefficient $k_{\mathrm{V}, \mathrm{B}} \in[0,1]$ describes the distribution of $\mathrm{B}$ from the feed to the primary product $V$ as

$$
y_{\mathrm{B}}=k_{\mathrm{V}, \mathrm{B}} z_{\mathrm{B}},
$$

with $y_{i}$ being molar fractions of components $i=\mathrm{A}, \mathrm{B}$ in the $V$ phase and $z_{i}$ being molar fractions in the feed. Since the secondary component B is supposed to be removed from the primary product $V, k_{\mathrm{V}, \mathrm{B}}$ can also be interpreted as an impurity distribution coefficient with regard to the primary product. In other 
words, better separation is achieved with lower values of $k_{\mathrm{V}, \mathrm{B}}$.

Molar fractions in the $L$ phase are denoted by $x_{i}$. The molar fraction $x_{\mathrm{B}}$ is determined by mass conservation $F z_{\mathrm{B}}=V y_{\mathrm{B}}+L x_{\mathrm{B}}$ and the remaining molar fractions of primary component A by summation conditions $z_{\mathrm{B}}+z_{\mathrm{A}}=1$, $x_{\mathrm{B}}+x_{\mathrm{A}}=1$ and $y_{\mathrm{B}}+y_{\mathrm{A}}=1$.

Specifying yield $Y_{\mathrm{V}}$ and distribution coefficient $k_{\mathrm{V}, \mathrm{B}}$ fully determines the product flows and purities of a single separation unit, which allows for comparing different separation processes in terms of these quantities. Other quantities such as the recovery of one component in one of the products and the purity of that product may also be used. Some useful relations to calculate other quantities from $Y_{\mathrm{V}}$ and $k_{\mathrm{V}, \mathrm{B}}$ are given in Appendix C.

With the definitions of yield and distribution coefficient in mind, the following equations are used for each separation unit.

$$
\begin{aligned}
V & =Y_{\mathrm{V}} F \\
V_{\mathrm{B}} & =k_{\mathrm{V}, \mathrm{B}} Y_{\mathrm{V}} F_{\mathrm{B}} \\
L & =F-V \\
L_{\mathrm{B}} & =F_{\mathrm{B}}-V_{\mathrm{B}}
\end{aligned}
$$

Note that depending on the model for the underlying separation process, using mass fractions instead of molar fractions for $x, y$ and $z$ and mass flows instead of molar flows for $F, V$ and $L$ might also be suitable.

\subsection{Superstructure for multistage separation}

The superstructure model is supposed to comprise all meaningful configurations for binary multistage separation processes while also excluding as many unsuitable configurations as possible to keep the numerical effort low. A separation network with $n=1, \ldots, N$ stages numbered downwards, as illustrated for three stages in Figure 2 is considered. Each stage $n$ separates a feed flow $F_{n}=F_{\mathrm{A}, n}+F_{\mathrm{B}, n}$ into primary product $V_{n}=V_{\mathrm{A}, n}+V_{\mathrm{B}, n}$ and secondary product $L_{n}=L_{\mathrm{A}, n}+L_{\mathrm{B}, n}$, with primary component $\mathrm{A}$ and secondary component $\mathrm{B}$. The feed $F_{n}$ of each stage $n$ is the sum of all primary products $V_{l}$ and secondary 


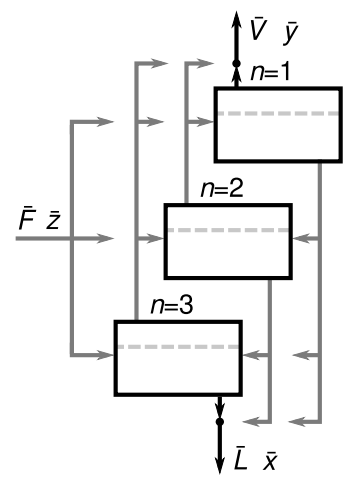

Figure 2: Superstructure for general multistage separation processes.

products $L_{l}, l=1, \ldots, N$, connected to that stage and, if applicable for that stage, the overall feed of the separation network $\bar{F}$.

All connections are implemented using binary variables $\beta \in\{0,1\}$. The variable $\beta_{n}^{\overline{\mathrm{F}}}$ attains a value of 1 if and only if the feed enters at stage $n$. Analogously, $\beta_{l, n}^{\mathrm{V}}, \beta_{l, n}^{\mathrm{L}}, \beta_{l}^{\overline{\mathrm{V}}}$ and $\beta_{l}^{\overline{\mathrm{L}}}$ attain a value of 1 if and only if the primary product of stage $l$ is fed to stage $n$, the secondary product of stage $l$ is fed to stage $n$, the primary product of stage $l$ is collected for the overall primary product of the separation network $\bar{V}$ and the secondary product of stage $l$ is collected for overall secondary product $\bar{L}$, respectively.

The resulting mass balance equations for the feed of each stage $n$ are

$$
\begin{aligned}
& F_{n}=\bar{F} \beta_{n}^{\overline{\mathrm{F}}}+\sum_{l=1}^{N} V_{l} \beta_{l, n}^{\mathrm{V}}+\sum_{l=1}^{N} L_{l} \beta_{l, n}^{\mathrm{L}}, \\
& F_{\mathrm{B}, n}=\bar{F}_{\mathrm{B}} \beta_{n}^{\overline{\mathrm{F}}}+\sum_{l=1}^{N} V_{\mathrm{B}, l} \beta_{l, n}^{\mathrm{V}}+\sum_{l=1}^{N} L_{\mathrm{B}, l} \beta_{l, n}^{\mathrm{L}} .
\end{aligned}
$$

The overall products of the separation network are calculated as

$$
\begin{aligned}
\bar{V} & =\sum_{l=1}^{N} \beta_{l}^{\overline{\mathrm{V}}} V_{l}, & \bar{V}_{\mathrm{B}} & =\sum_{l=1}^{N} \beta_{l}^{\overline{\mathrm{V}}} V_{\mathrm{B}, l}, \\
\bar{L} & =\sum_{l=1}^{N} \beta_{l}^{\overline{\mathrm{L}}} L_{l}, & \bar{L}_{\mathrm{B}} & =\sum_{l=1}^{N} \beta_{l}^{\overline{\mathrm{L}}} L_{\mathrm{B}, l} .
\end{aligned}
$$

Following relations are used for molar fractions at the feed of the separation network $\bar{z}_{\mathrm{B}}$, the overall primary product of the separation network $\bar{y}_{\mathrm{B}}$ and the 
overall secondary product $\bar{x}_{\mathrm{B}}$.

$$
\bar{F}_{\mathrm{B}}=\bar{z}_{\mathrm{B}} \bar{F}, \quad \bar{L}_{\mathrm{B}}=\bar{x}_{\mathrm{B}} \bar{L}, \quad \bar{V}_{\mathrm{B}}=\bar{y}_{\mathrm{B}} \bar{V}
$$

The following relations ensure that the feed as well as each product of any stage is connected to only one target. Note that in the present study stream splitting is not considered for the superstructure.

$$
\sum_{l=1}^{N} \beta_{l}^{\overline{\mathrm{F}}}=1, \quad \sum_{l=1}^{N} \beta_{n, l}^{\mathrm{V}}+\beta_{n}^{\overline{\mathrm{V}}}=1, \quad \sum_{l=1}^{N} \beta_{n, l}^{\mathrm{L}}+\beta_{n}^{\overline{\mathrm{L}}}=1
$$

Equally, each stage is required to have some connection to its feed:

$$
\sum_{l=1}^{N}\left(\beta_{l, n}^{\mathrm{V}}+\beta_{l, n}^{\mathrm{L}}\right)+\beta_{n}^{\overline{\mathrm{F}}} \geq 1
$$

Since the primary component is selectively concentrated in the primary product, the primary product of each stage is only transported in the direction of the overall primary product of the separation network in order to avoid backmixing. The secondary product is treated in the same manner:

$$
\begin{array}{lll}
\beta_{l, n}^{\mathrm{V}}=0 & \text { for all } & 1 \leq l \leq n \\
\beta_{l, n}^{\mathrm{L}}=0 & \text { for all } & n \leq l \leq N
\end{array}
$$

Finally, the stages are ordered in terms of the molar fraction of the secondary component in the feed $z_{\mathrm{B}, n} \leq z_{\mathrm{B}, n+1}$, which is implemented using flow rates that are available from the model equations above.

$$
F_{\mathrm{B}, n} F_{n+1} \leq F_{\mathrm{B}, n+1} F_{n}, \quad n=1, \ldots, N-1
$$

\subsection{Separation effort}

Economical cost determination requires many decisions about the actual implementation of a process and introduces significant uncertainties due to heuristic cost estimates, both of which make it difficult to interpret optimization results and draw general conclusions from it.

In the present work, a general performance indicator is chosen that allows to 
increase process understanding based on optimization results and provide additional options and guidelines for subsequent economical process design. These guidelines extend available knowledge at early design steps and do not replace steps with more detailed process and cost models.

The effort for separation processes usually scales with the amount of processed product phase, e.g. energy consumption and vessel size for filtration units strongly scale with the amount of permeate, and for crystallization units with the crystal mass. The separation effort, i. e. the ratio of the total amount of primary product for all stages to the overall amount of feed, therefore gives a measure of the operating and investment costs of a process. The separation effort $J$ as used in the following studies is defined by

$$
J=\frac{\sum_{l=1}^{N} V_{l}}{\bar{F}} .
$$

Optimization results using the separation effort were shown to be similar to economical optimization results for the conceptual design of cooling crystallization in [26, 39]. Furthermore, similar objective functions are used in the literature. The crystallization effort defined in [2, 40] for cooling crystallization

135 as the total amount of crystallized mass divided by product mass is equivalent to the separation effort for fixed feed and product specifications. The evaporation effort, introduced in [8] for evaporative crystallization, is defined as the total amount of evaporated solvent per feed amount and scales monotonically with the separation effort. The idea of rating the reprocessing of products for process evaluation is extended in the current work to filtration processes.

\section{Process models}

\subsection{Cooling crystallization}

Cooling crystallization for the separation of a eutectic two-component mixture without an additional dedicated solvent is adapted from [5]. Layer crystallization is a specific technique where the mixture flows over a cooled surface on which a solid crystal layer is formed. Remaining liquid is drained from the solid 
layer to separate the product phases. The primary product of the process is the solid layer denoted by $V$ and the secondary product is the remaining liquid $L$. Crystallization of eutectic mixtures as depicted in Figure 3 ideally produces pure solid of a type determined by the position of the initial liquid composition relative to the eutectic point. However, liquid inclusions and adhering liquid lead

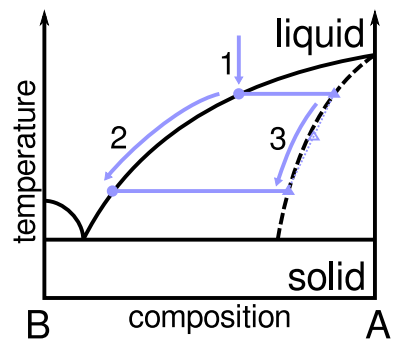

Figure 3: Example phase equilibrium for eutectic mixture. Cooling crystallization: (1) cooling, crystals forming at liquidus line. (2) changing liquid composition due to selective removal of A. (3) crystal impurities due to inclusion of liquid.

to practical impurity of the solid layer. The purity of the solid layer may be increased by additional measures like washing and sweating. Since the eutectic point can not be crossed, feasible product compositions are limited. Handling of solids can be avoided by melting the the solid layer again. While the equipment for layer crystallization is relatively simple, sufficient crystal growth rates often require large cooling surfaces. For more details on this type of process we refer to [41].

The separation efficiency for this process is given by the differential distribution coefficient $k_{\text {diff }}$, which describes the ratio of the amount of secondary component to the total amount added to the solid layer relative to the fraction of secondary component in the liquid. In short, $k_{\mathrm{diff}}$ is a measure for the local impurity distribution from liquid to solid. Since the liquid composition changes during layer growth due to selective removal of the primary component, the local composition of the solid layer varies with layer thickness. The mean composition of the solid layer is thus determined by the differential distribution coefficient $k_{\text {diff }}$ as well as the yield of primary product $Y_{\mathrm{V}}$. The overall distribution coefficient of 
the secondary component to the primary product $k_{\mathrm{V}, \mathrm{B}}$ has to be calculated via integration. The differential distribution coefficient is an aggregated parameter that incorporates several operational variables such as crystal growth rate and liquid flow properties. It is assumed here that $k_{\text {diff }}$ is constant and equal for all units in a crystallization network. The resulting model for the distribution coefficient of the secondary component to the primary product $k_{\mathrm{V}, \mathrm{B}}$ is

$$
k_{\mathrm{V}, \mathrm{B}}=\frac{1-\left(1-Y_{\mathrm{V}}\right)^{k_{\text {diff }}}}{Y_{\mathrm{V}}}
$$

with liquid molar fraction of $\mathrm{B}$ restricted by $x_{\mathrm{B}}^{\max }$ due to the eutectic point:

$$
x_{\mathrm{B}}^{\max } L \geq L_{\mathrm{B}}
$$

As depicted in Figure 4 the highest separation efficiency for this process, or lowest value of distribution coefficient $k_{\mathrm{V}, \mathrm{B}}$, is achieved for zero yield at $k_{\mathrm{V}, \mathrm{B}}\left(Y_{\mathrm{V}}=0\right)=k_{\text {diff }}$. The separation efficiency decreases with increasing yield up to a point where the liquid composition approaches the eutectic composition.

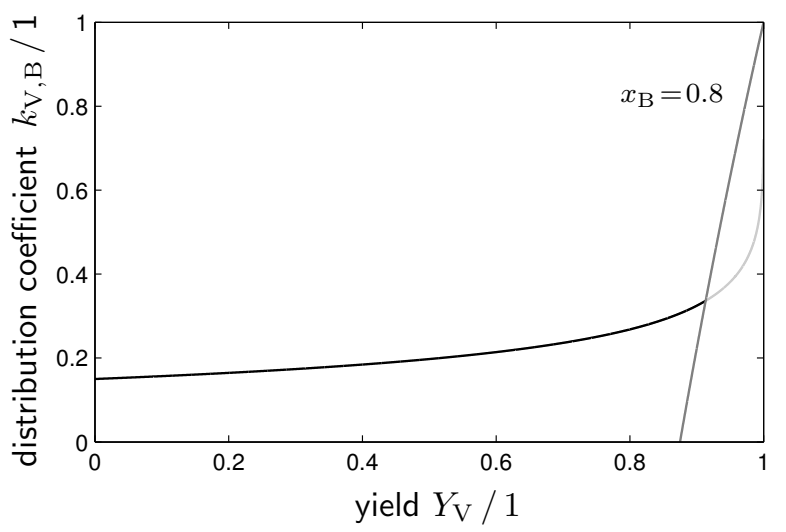

Figure 4: Cooling crystallization. Distribution of impurity to product depending on yield. Example for $k_{\text {diff }}=0.15$. The dark gray line depicts where the composition of the residual liquid reaches an exemplary maximum value of $x_{\mathrm{B}}=0.8$ for a feed composition of $z_{\mathrm{B}}=0.1$. 


\subsection{Filtration}

A process for organic solvent nanofiltration using the solution-diffusion model for permeation through the membrane as in 42 is studied here. A pressure difference between the two sides of a dense membrane causes a diffusive flow through the membrane. The resulting flow is influenced by the permeabilities $k_{\mathrm{P}, i}$ of participating components $i$. The permeability is an aggregated parameter that comprises the geometry of the membrane and the interaction of components with the membrane material. A high permeability results in a large permeate flow of the corresponding component, while large differences between components lead to an increased concentration of the component with higher permeability in the permeate flow. An advantage of filtration processes is that they do not require phase changes. However, the availability of suitable membrane materials and membrane fouling are possible disadvantages.

A one-dimensional setup for the flow through the unit as depicted in Figure 5 is considered here. The primary product of the filtration process is the permeate $V$

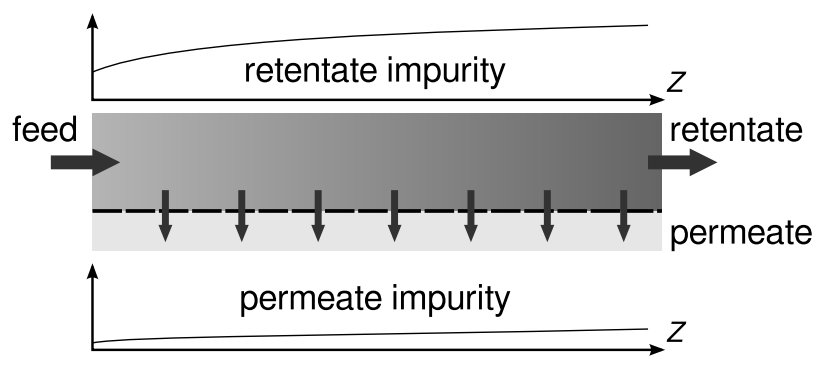

Figure 5: Filtration unit.

and the secondary product is the retentate $L$. The local flow through the membrane depends on the liquid composition at the membrane, the temperature and the pressure difference, whereas the total permeate flow of a membrane unit is integrated over the total membrane area using a suitable discretization scheme. The following additional assumptions are made to reduce computational cost and allow analyzing configurations of multiple membrane units:

- plug flow on feed side, 
- ideal mixing perpendicular to membrane surface on feed side,

- no mixing in flow direction on feed side,

- no sweep flow on permeate side,

- constant and equal pressure difference and temperature in all units.

Analogously to the cooling crystallization described above, these assumptions leave the primary product yield $Y_{\mathrm{V}}$ represented by the membrane area as the single degree of freedom for each membrane unit. As depicted in Figure 6 the highest separation efficiency is achieved for zero yield $Y_{\mathrm{V}}=0$ and decreases with increasing yield up to no separation at $Y_{\mathrm{V}}=1$. The separation character-

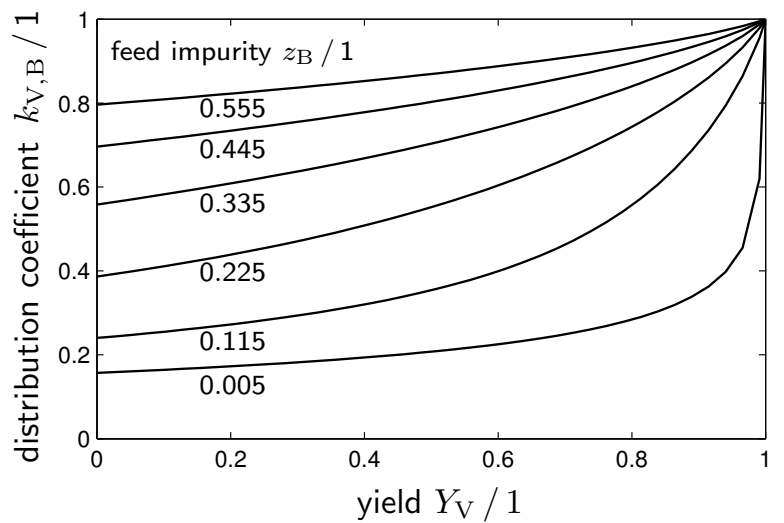

Figure 6: Filtration. Distribution of impurity to product depending on yield for different feed impurities. Lower distribution coefficient for lower feed impurity. 50 discretization elements per stage.

istics for filtration with fixed feed composition are similar to those of cooling crystallization. In fact, the separation characteristic of nanofiltration with the concentration of the secondary component approaching zero is identical to a cooling crystallization process with

$$
k_{\mathrm{diff}}=\frac{k_{\mathrm{P}, \mathrm{B}}}{k_{\mathrm{P}, \mathrm{A}}\left(1-\exp \left(-\frac{\nu_{\mathrm{A}} \Delta P}{\mathrm{R} T}\right)\right)+k_{\mathrm{P}, \mathrm{B}} \exp \left(-\frac{\nu_{\mathrm{B}} \Delta P}{\mathrm{R} T}\right)} .
$$

Here, $\nu_{\mathrm{i}}$ are molar volumes, $\Delta P$ is the pressure difference between both sides of 175 the membrane, $T$ is the temperature and $\mathrm{R}$ is the ideal gas constant. 
The complete model equations for the organic solvent nanofiltration process are given in Appendix A.

\subsection{Evaporative crystallization}

Mixtures of components that form a homogeneous solid phase are called solid solutions. Due to this property solid solutions usually require multiple steps for separation via crystallization. Multistage cascades for the separation of solid solutions have been studied in e. g. [6, 7, 9]. The model for evaporative crystallization of a two-component mixture forming a solid solution used in the present work is adapted from $[8$.

The process for evaporative crystallization is shown in Figure 7. Any solid contained in the feed of a crystallization unit is dissolved by adding solvent. Supersaturation and crystal growth is induced by removing solvent through evaporation. When the desired solid mass is achieved the remaining liquid is drained to separate the product phases. The primary product is the solid $V$ and the secondary product is the liquid $L$. Including the solvent results in a

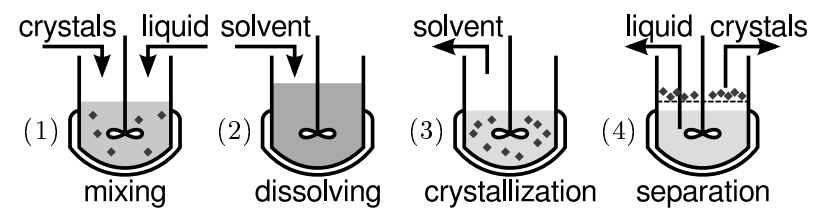

Figure 7: Evaporative crystallization. Process steps.

three-component mixture instead of the two-component mixtures considered in the previous sections. However, all relations introduced previously for general separation units and multistage separation networks still hold. The following equations are added to the model of the separation network to account for transport of solvent $\mathrm{S}$ between units:

$$
\begin{aligned}
& F_{\mathrm{S}, n}=\bar{F}_{\mathrm{S}} \beta_{n}^{\overline{\mathrm{F}}}+\sum_{l=1}^{N} V_{\mathrm{S}, l} \beta_{l, n}^{\mathrm{V}}+\sum_{l=1}^{N} L_{\mathrm{S}, l} \beta_{l, n}^{\mathrm{L}}, \\
& \bar{V}_{\mathrm{S}}=\sum_{l=1}^{N} \beta_{l}^{\overline{\mathrm{V}}} V_{\mathrm{S}, l}, \quad \bar{L}_{\mathrm{S}}=\sum_{l=1}^{N} \beta_{l}^{\overline{\mathrm{L}}} L_{\mathrm{S}, l} .
\end{aligned}
$$


The separation efficiency for this process is determined by the phase equilibrium (see Figure 8 for a mixture of potassium sulfate, ammonium sulfate and water). Corresponding compositions of solid and saturated liquid show how much the concentration of the primary component relative to the secondary component is increased in the solid phase. The phase equilibrium is temperature dependent. Assuming that the temperature is constant and equal for all crystallization units

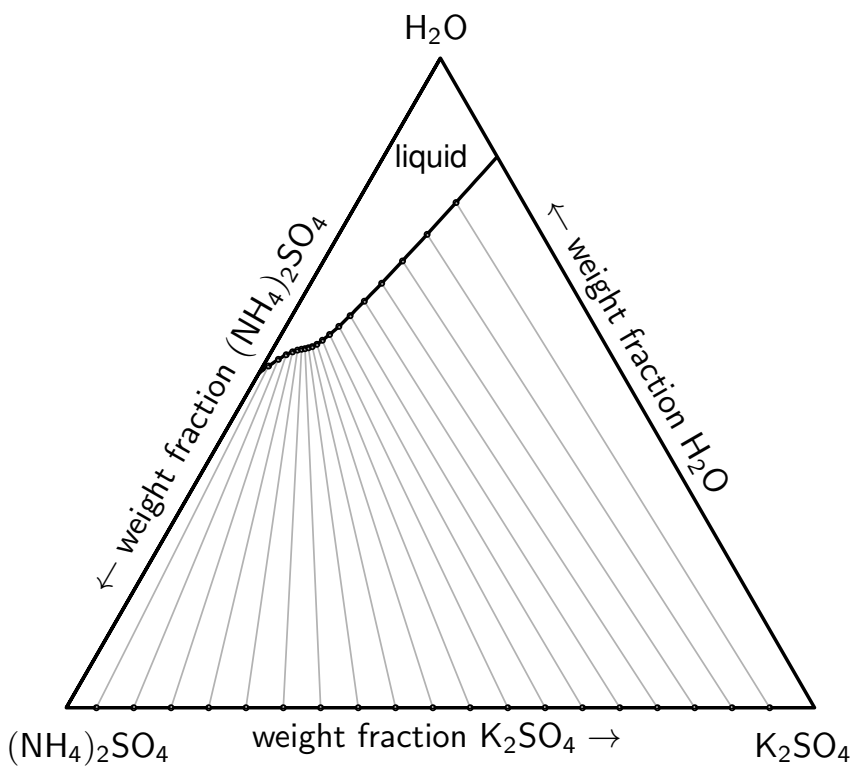

Figure 8: Phase diagram of potassium sulfate, ammonium sulfate and water at $65^{\circ} \mathrm{C}$, with potassium sulfate and ammonium sulfate forming solid solutions. Corners represent pure components and edges binary mixtures. Gray lines depict corresponding equilibrium compositions in the solid and liquid phase. Model from [8].

leaves the primary product yield $Y_{\mathrm{V}}$, represented by the amount of solvent that needs to be vaporized, as the single degree of freedom for each crystallization unit. As depicted in Figure 9 the highest separation efficiency is achieved for zero yield $Y_{\mathrm{V}}=0$ and decreases with increasing yield up to no separation at $Y_{\mathrm{V}}=1$. A performance indicator for evaporative crystallization $J$ is given by the overall amount of evaporated solvent relative to the feed and comprises two 


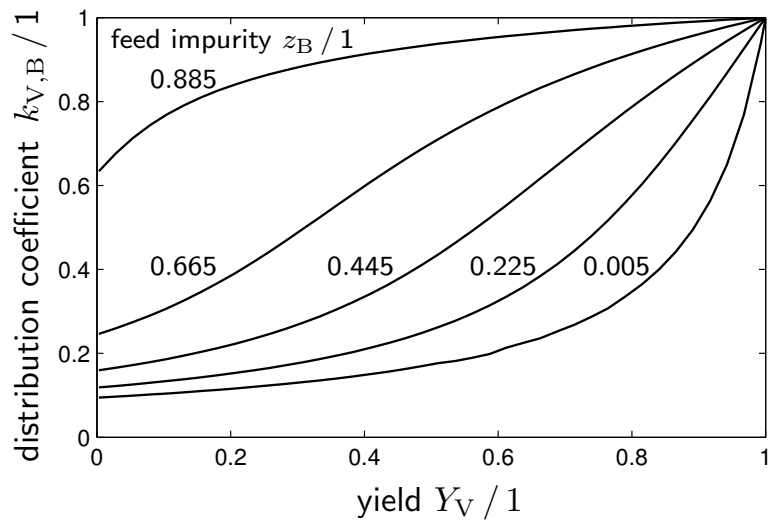

Figure 9: Evaporative crystallization. Distribution of impurity to product depending on yield for different feed impurities. Lower distribution coefficient for lower feed impurity.

separate parts:

$$
J=\frac{\sum_{n=1}^{N} S_{n}}{\bar{F}}+\frac{\bar{V}_{\mathrm{S}}+\bar{L}_{\mathrm{S}}}{\bar{F}} .
$$

The evaporated solvent for all units $\frac{\sum_{n=1}^{N} S_{n}}{\bar{F}}$ corresponds to the general separation effort defined in (17). The solvent that has to be removed from the overall products of the separation network $\frac{\bar{V}_{S}+\bar{L}_{S}}{\bar{F}}$ is a constant term for fixed feed and product specifications and therefore does not influence the solution of the optimization problem. However, it limits any potential reduction of the objective value.

185 As an additional difference, the evaporated solvent is not directly proportional to the primary product but determined by the solubility line. The relation between the evaporated solvent and the primary product of a unit depending on the yield and feed composition is depicted in Figure 10 . The complete model equations are reported in Appendix B. 


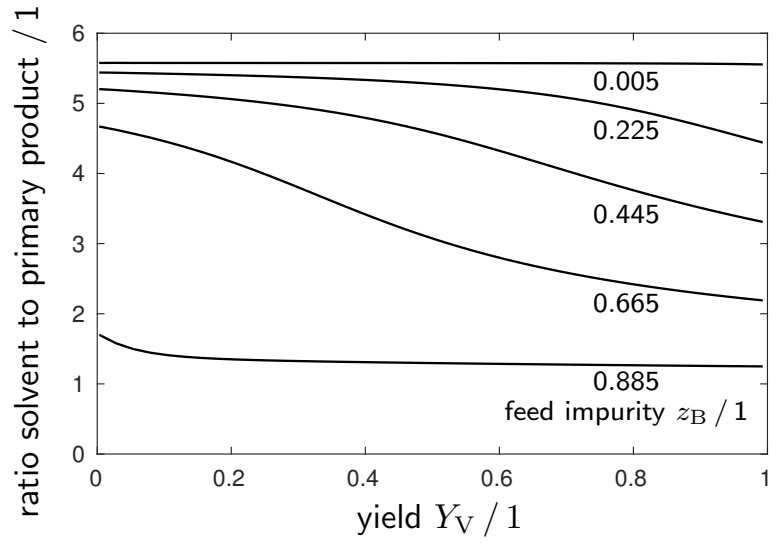

Figure 10: Evaporative crystallization. Ratio of evaporated solvent to primary product depending on yield for different feed impurities.

\section{Definition of optimization problem}

The following studies require solving problems of the type

$$
\begin{array}{ll}
\min & J(\boldsymbol{x}, \boldsymbol{y}), \\
\text { s.t. } & \boldsymbol{f}(\boldsymbol{x}, \boldsymbol{y}, \boldsymbol{p})=\mathbf{0}, \\
& \boldsymbol{g}(\boldsymbol{x}, \boldsymbol{y}, \boldsymbol{p}) \leq \mathbf{0}, \\
& \boldsymbol{x} \in X, \quad X \subset \mathbb{R}^{N_{\mathrm{x}}}, \\
& \boldsymbol{y} \in\{0,1\}^{N_{\mathrm{y}}},
\end{array}
$$

with objective function $J$, equality constraints $f$ such as mass balances, inequality constraints $\boldsymbol{g}$ such as minimum product purities, real numbered variables $\boldsymbol{x}$ such as mass flows with according set $X$ and binary variables $\boldsymbol{y}$ for the modeling of connections between stages. The parameter vector $\boldsymbol{p}$ is varied to study the sensitivity of optimal configurations to parameter values. The resulting mixed-integer nonlinear problems (MINLPs) are difficult to solve due to the existence of suboptimal local solutions. Globally optimal solutions are determined by applying the branch-and-bound-type solver BARON [43], with subsolvers CONOPT and CPLEX. The termination tolerances are chosen as optca $=0$ and optcr $=0.0001$. 


\section{Results}

\subsection{Tapered structures}

A common candidate configuration for multistage separation processes is illustrated in Figure 11. In this so-called tapered structure the secondary product

of each stage is the feed of the subsequent stage. The secondary product of the last stage is collected as the overall secondary product. The primary product of each stage is collected for the overall primary product of the separation network. Also, each stage in Figure 11 may comprise a number of parallel units. It can be

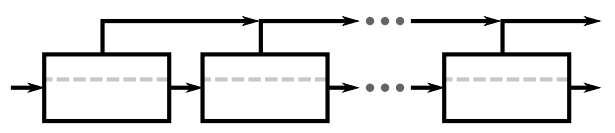

Figure 11: Tapered structure.

shown that a single separation unit is equivalent to a two-stage tapered configuration in terms of product specification and separation effort under following conditions.

- identical yield of secondary product:

$$
Y_{\mathrm{L}}=Y_{\mathrm{L}, 1} Y_{\mathrm{L}, 2}
$$

- identical composition of secondary product:

$$
k_{\mathrm{L}, \mathrm{B}}\left(Y_{\mathrm{L}}\right)=k_{\mathrm{L}, \mathrm{B}}\left(Y_{\mathrm{L}, 1}\right) k_{\mathrm{L}, \mathrm{B}}\left(Y_{\mathrm{L}, 2}\right)
$$

Here, $Y_{\mathrm{L}}$ is the secondary product yield of a single unit with distribution coefficient of secondary component to secondary product $k_{\mathrm{L}, \mathrm{B}}$ and $Y_{\mathrm{L}, 1 / 2}$ are the secondary product yields of two units connected in a tapered structure.

Validity of these conditions implies that for each multistage configuration there exist any number of equivalent configurations where one or more stages are replaced by a tapered structure of stages. To get only the configuration with the lowest number of stages, tapered structures have to be excluded in that case. It is important to note that performance limiting effects may limit the maximum 
size or throughput of single units in an actual implementation of a separation process. This includes concentration polarization as well as bounds for the flow rate and the pressure drop in membrane modules [27, 31, but is not limited to membrane processes. Separation modules connected in a tapered structure can be used to overcome such limitations and increase the size of a separation stage in an appropriate way [30.

Furthermore, for

$$
k_{\mathrm{L}, \mathrm{B}}\left(Y_{\mathrm{L}}\right)>k_{\mathrm{L}, \mathrm{B}}\left(Y_{\mathrm{L}, 1}\right) k_{\mathrm{L}, \mathrm{B}}\left(Y_{\mathrm{L}, 2}\right),
$$

tapered structures are better than single stages in terms of separation efficiency, which also means that they offer less separation effort for the same separation results. Since the best configurations are only approached for infinite-stage tapered structures, configurations containing tapered structures should be discussed separately from other optimization results.

The distribution coefficient of the secondary component to the secondary product $k_{\mathrm{L}, \mathrm{B}}$ is evaluated using $k_{\mathrm{V}, \mathrm{B}}$ with

$$
k_{\mathrm{L}, \mathrm{B}}=\frac{1-k_{\mathrm{V}, \mathrm{B}}\left(Y_{\mathrm{V}}\right) Y_{\mathrm{V}}}{1-Y_{\mathrm{V}}}, \quad Y_{\mathrm{V}}=1-Y_{\mathrm{L}} .
$$

If the distribution coefficient additionally depends on the feed composition as $k_{\mathrm{L}, \mathrm{B}}\left(Y_{\mathrm{L}}, z_{\mathrm{B}}\right)$, the relations

$$
z_{\mathrm{B}, 1}=z_{\mathrm{B}}, \quad z_{\mathrm{B}, 2}=k_{\mathrm{L}, \mathrm{B}}\left(Y_{\mathrm{L}, 1}, z_{\mathrm{B}}\right)
$$

have to be included.

\subsection{Cooling crystallization}

Model parameters affecting the optimal configuration of a crystallizer network are the differential distribution coefficient $k_{\text {diff }}$, the feed composition $\bar{z}$ and the product specifications $\bar{y}, \bar{x}$. The eutectic composition is implicitly included in this set of parameters by limiting the feasible range for the feed composition and product specifications. 
A comprehensive characterization of optimal solutions for the considered crystallization model is given using parameter studies for $k_{\text {diff }}$ and $\bar{z}$, representing the selectivity of the process and the initial composition of the mixture. Changing the product requirements was found to provide no additional qualitative properties of optimal solutions and corresponding results are therefore not included here. Note that changing the selectivity corresponds to considering different types of mixtures. Parameter values and domain specifications for the following parameter study are reported in Table 1 .

Optimization is performed for each parameter combination $\left(k_{\mathrm{diff}}, \bar{z}_{\mathrm{B}}, N\right)$ of differential distribution coefficient, feed composition and number of stages. The conditions for tapered structures (25) and (26) are valid for the cooling crystallization model. Accordingly, redundant configurations containing tapered structures are removed from the optimization results.

In the following figures, each differently colored region represents a different globally optimal configuration of a crystallizer network, i.e. at least one structure variable $\beta$ assumes a different value. White dots represent sets of parameters for which a global optimum is determined. The content of secondary component in the feed is normalized to the product specifications according to

$$
\bar{z}_{\text {norm }}=\frac{\bar{z}_{\mathrm{B}}-\mathrm{up}\left(\bar{y}_{\mathrm{B}}\right)}{\operatorname{lo}\left(\bar{x}_{\mathrm{B}}\right)-\operatorname{up}\left(\bar{y}_{\mathrm{B}}\right)},
$$

with $\bar{z}_{\text {norm }} \in[0,1]$.

Results for optimal countercurrent cascades are given as a reference for optimal configurations based on the general superstructure from section 2.2 Values of binary variables are restricted according to equations (31)-(34) to limit the search space to countercurrent cascades.

$$
\begin{aligned}
& \beta_{l, l-1}^{\mathrm{V}}=1, \quad l=2, \ldots, N \\
& \beta_{l, l+1}^{\mathrm{L}}=1, \quad l=1, \ldots, N-1 \\
& \beta_{1}^{\overline{\mathrm{V}}}=1 \\
& \beta_{N}^{\overline{\mathrm{L}}}=1
\end{aligned}
$$


Resulting parameter regions for different optimal countercurrent cascades are depicted in Figure 12. The configuration of a countercurrent cascade is fully

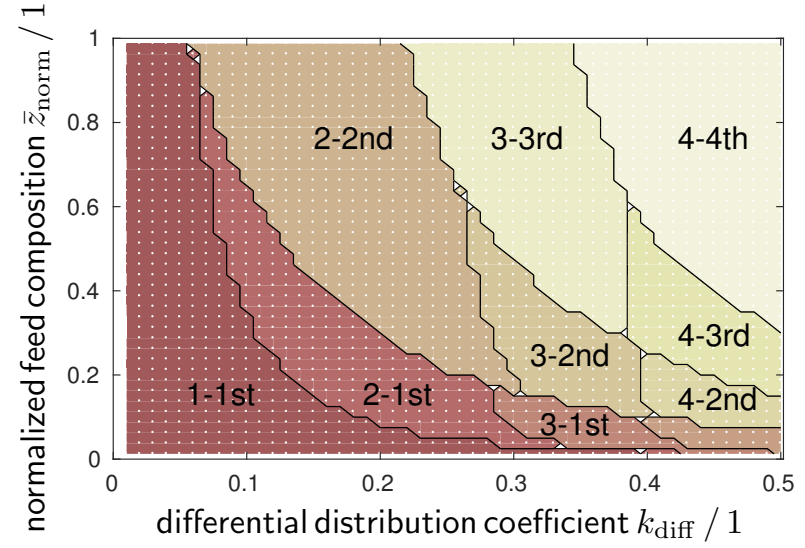

Figure 12: Cooling crystallization. Globally optimal configuration of countercurrent crystallizer cascade defined by number of stages and feed stage. Each patch represents a configuration with a different combination of "number of stages - feed stage".

characterized by the number of stages and the feed stage position. Sufficiently low product requirements (i.e. product specifications close to the feed composition) and high separation efficiency (i. e. small values for the differential distribution coefficient) allow separation of a mixture in one crystallization step. This case is represented by the lower left region of Figure 12. With increasing secondary component content in the feed and decreasing separation efficiency, more separation steps are required. Accordingly, the optimal number of stages increases with increasing normalized feed concentration and distribution coefficient, up to four stages in the upper right region of Figure 12. The separation effort as used in this work is minimized by minimizing the sum of primary product flows over all stages. If the feed enters the first stage, counted from the top, the amount of overall primary product has to be crystallized at least once. If the feed enters the second stage of a countercurrent cascade the overall primary product has to be crystallized at least twice, and so on, potentially increasing the separation effort. However, larger composition differences between feed and feed stage require larger recycle flows, making higher feed stage numbers 
more favorable for large secondary component content in the feed and lower separation efficiency. The resulting optimal feed position is on the first stage, counted from the top, for low secondary component content in the feed and high separation efficiency, as in the lower left region in Figure 12 . The optimal feed stage number increases with increasing secondary component content in the feed and increasing distribution coefficient, up to the fourth stage in the upper right region of Figure 12 .

Results for the general superstructure from section 2.2 are discussed below. In Figure 13 the separation effort for optimal configurations from the general superstructure is compared to that for countercurrent cascades. Outlines of the parameter regions from Figure 12 are included in Figure 13 as a reference frame. Optimal configurations with lower separation effort are located near boundaries

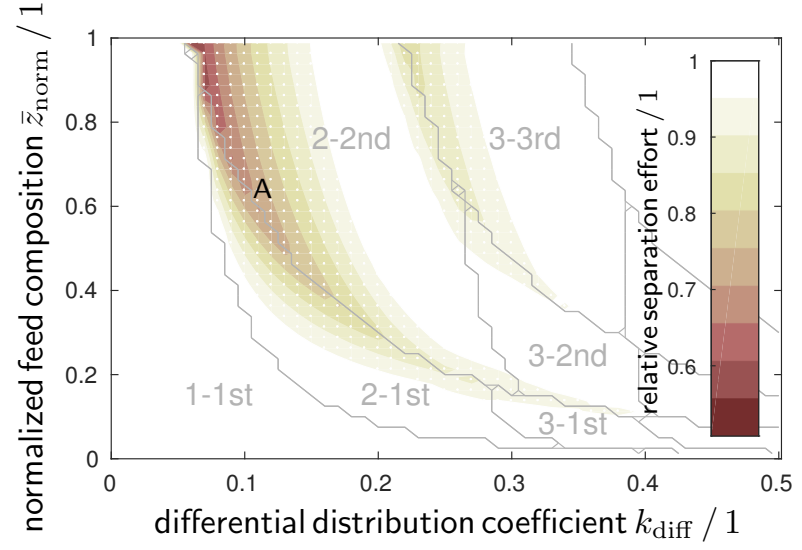

Figure 13: Cooling crystallization. Ratio of separation effort for optimal general configurations to separation effort for countercurrent cascades. Grey lines indicate parameter regions of Figure 12 with "number of stages - feed stage" of according countercurrent cascades.

of parameter regions with different countercurrent feed positions. The largest improvements are found in the boundary region of "2-1st" and "2-2nd" of Figure 12 i.e. where optimal countercurrent cascades have two stages. There are actually three different configurations in that region that have the same optimal separation effort, depicted in Figure 14. All three configurations have the same feed positions, i. e. values of $\beta_{l}^{\overline{\mathrm{F}}}$, and and main product connections, i.e. values 
(a)

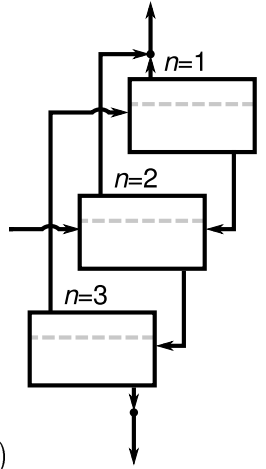

(b)

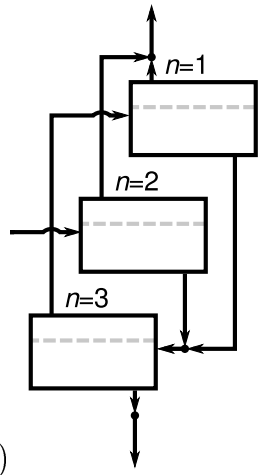

(c)

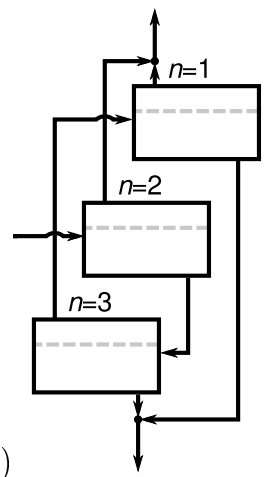

Figure 14: Cooling crystallization. Equivalent globally optimal configurations for region A in Figure 13

common mechanism for all three configurations is explained as follows. The feed stage generates primary product with purity below specification. The remaining two stages are used to generate primary product with purity above specification from the secondary product of the feed stage. The primary products are mixed together to exactly meet the product specification. Using this structure avoids crystallizing the whole primary product amount twice, as is the case for countercurrent configurations with two stages and the second stage as feed position. It also avoids large recycles from a stripping stage in the case of the first stage being the feed position and the feed composition being far from the product specification. Instead, there is a "pre-treatment" stage that removes as much primary product as possible below the purity specification such that the specification is still met by mixing with primary product from two crystallizer stages in series. Selected optimal designs from region A in Figure 13 are given in detail in the supplementary information.

Note that these results mirror previous results using an economical objective function in 26] due to that economical objective function depending monotonically on the separation effort. Independent baseline investment costs lead to lower reductions of economical cost than separation effort, with observed values 
for potential reduction of economical cost.

\subsection{Filtration}

Parameter studies analogous to those in the previous section are carried out for an organic solvent nanofiltration process. Parameter values are taken from 44. for a mixture of decane (main component A) and hexacosane (secondary component B). Concentrations of hexacosane in the following parameter study are limited to the same range of small values as in the experiments carried out in [44].

In the same way as in the previous section, the selectivity of the process and the feed composition are varied to study their influence on globally optimal multistage filtration networks. The selectivity is varied by changing the value of the permeability coefficient of the secondary component $k_{\mathrm{P}, \mathrm{B}}$. Decreasing this value decreases permeation of the secondary component $B$, thereby increasing selectivity of the desired permeation product A. In practice, this could be achieved by changing the interaction between the components and the membrane, e.g. by using a different membrane material or by considering a different mixture. The permeability coefficient $k_{\mathrm{P}, \mathrm{B}}$ is normalized to a reference value taken from [44] as

$$
k_{\mathrm{P}, \mathrm{B}, \mathrm{norm}}=\frac{k_{\mathrm{P}, \mathrm{B}}}{k_{\mathrm{P}, \mathrm{B}, \mathrm{ref}}} .
$$

The feed composition is normalized in the same way as in the previous section, see (30). Optimization for the general superstructure and for countercurrent configurations is performed for each parameter combination $\left(k_{\mathrm{P}, \mathrm{B}, \mathrm{norm}}, \bar{z}_{\text {norm }}, N\right)$. Parameter and domain specifications are given in Table 2. A small number of discretization points is chosen to keep the computational cost low. Results calculated with different numbers of discretization points indicate that the chosen value is sufficient for this study.

Tapered structures are equivalent to single membrane units for the considered process model and excluded from optimization results. 
The optimization results for organic solvent nanofiltration are shown in Figure 15. The results are equivalent to those for cooling crystallization discussed in the previous section. This is due to the fact that the separation characteristic for filtration with low content of secondary component in the feed is similar to that of cooling crystallization, compare Figure 6 and Figure 4 However, only configuration (b) in Figure 14 is globally optimal for the entire parameter region A in Figure 15 where optimal countercurrent cascades have two stages.

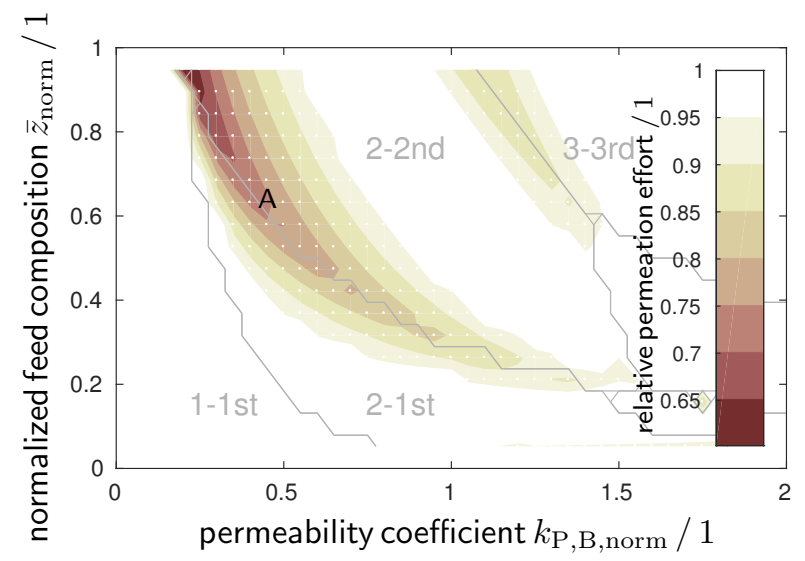

Figure 15: Filtration. Ratio of permeation effort for optimal general configurations to permeation effort for countercurrent cascades. Grey lines indicate parameter regions of optimal countercurrent configurations with "number of stages - feed stage".

\subsection{Evaporative crystallization}

Parameter influence on globally optimal configurations of evaporative crystallization units is studied for a mixture of potassium sulfate (main component A), ammonium sulfate (secondary component B) and water (solvent) at $65^{\circ} \mathrm{C}$ with parameter values taken from [8].

The influence of selectivity and feed composition on globally optimal solutions is studied by varying according parameter values. The selectivity is adjusted by scaling the equilibrium composition of the solid phase while keeping the solubility line unchanged, see Appendix B. Selectivity scaling is used here instead of considering a large number of mixtures with different selectivities. Values 
of the scaling parameter $k_{\alpha}$ smaller than one imply less secondary component content in the solid phase and therefore better selectivity. Values of $k_{\alpha}$ greater than one imply worse selectivity compared to that for the reference parameters. The scaled selectivity for the mixture considered here is depicted by the solid composition as a function of the liquid composition without solvent in Figure 16 for a range of values $k_{\alpha}$. The feed composition is normalized according to equa-

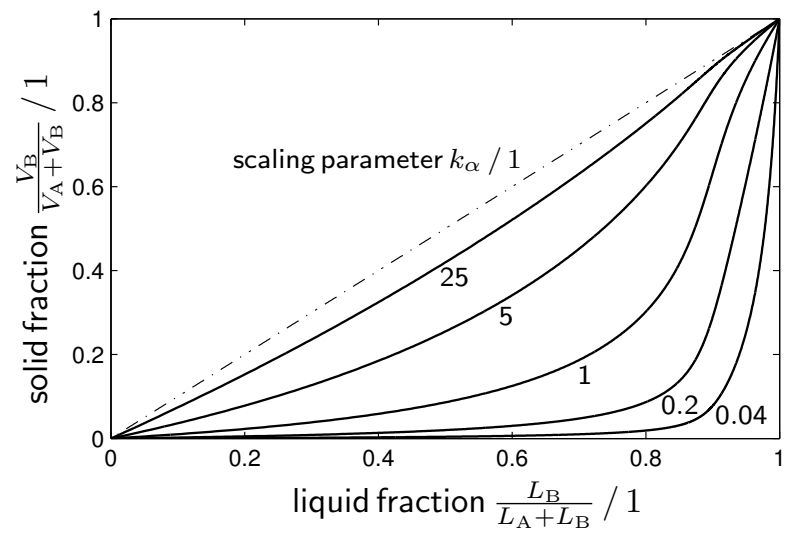

Figure 16: Evaporative crystallization. Selectivity of secondary component B between solid phase and liquid phase at equilibrium for different values of the selectivity scaling parameter $k_{\alpha}$.

tion (30). Optimization for the general superstructure and for countercurrent configurations is performed for each parameter combination $\left(k_{\alpha}, \bar{z}_{\text {norm }}, N\right)$. Parameter and domain specifications are given in table 3 .

Tapered structures for evaporative crystallization processes correspond to repeated crystallization with intermediate removal of the solid phase from the crystallization unit. Intermediate removal of the solid phase increases the separation efficiency by removing limitations imposed by equilibrium conditions. Therefore, the separation effort of any multistage configuration is reduced by replacing single stages with tapered structures.

The following results are generated by initial optimization using the general superstructure and subsequent optimization for only those optimal configurations identified in the first step that do not contain tapered structures. The 
evaporation effort without product drying $\frac{\sum_{n=1}^{N} S_{n}}{F}$ is used in Figure 17 instead of the objective $J$. This allows easier comparison of results with the other case studies. However, note that improved configurations provide less reduction of the objective $J$ with product drying included than the values shown in Figure 17. namely up to $18 \%$ reduction compared to optimal countercurrent cascades. The number of stages is limited to three. Improved configurations are thus only found compared to two-stage countercurrent cascades. The results for evaporative crystallization qualitatively match the results for cooling crystallization and filtration. The globally optimal configuration in region A, a parameter region where optimal countercurrent cascades have two stages, is the same as configuration (b) in Figure 14 for cooling crystallization and the same as for filtration. Configurations with less evaporation effort can be generated by

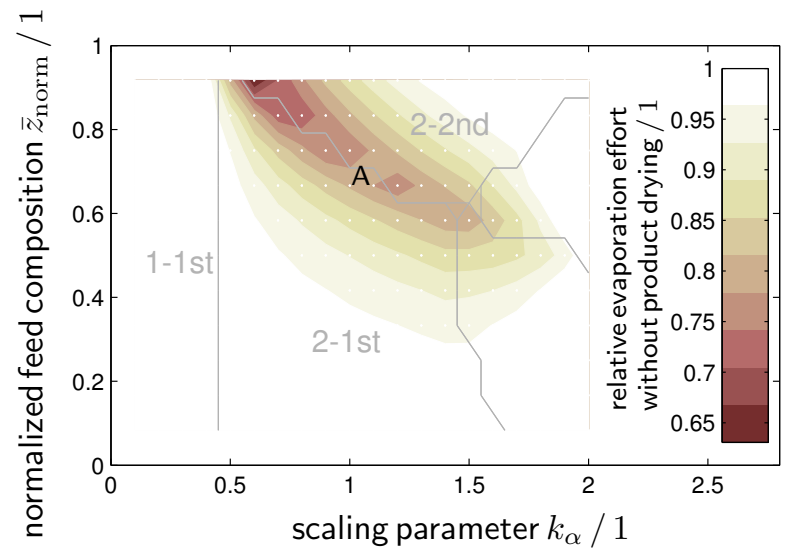

Figure 17: Evaporative crystallization. Ratio of evaporation effort without product drying $\left(\sum_{n=1}^{N} S_{n}\right) \bar{F}^{-1}$ for optimal general configurations to that for countercurrent cascades. Grey lines indicate parameter regions of optimal countercurrent configurations with "number of stages - feed stage".

replacing stages in known optimal configurations with tapered structures at the cost of increasing the number of stages. The biggest improvements are achieved by continuous removal of the solid phase, which is approximated by tapered structures with a large number of stages. Note that some technological implementation of single stage continuous removal of the solid phase might also be 
possible. The separation characteristics for a tapered structure with 50 stages are depicted in Figure 18

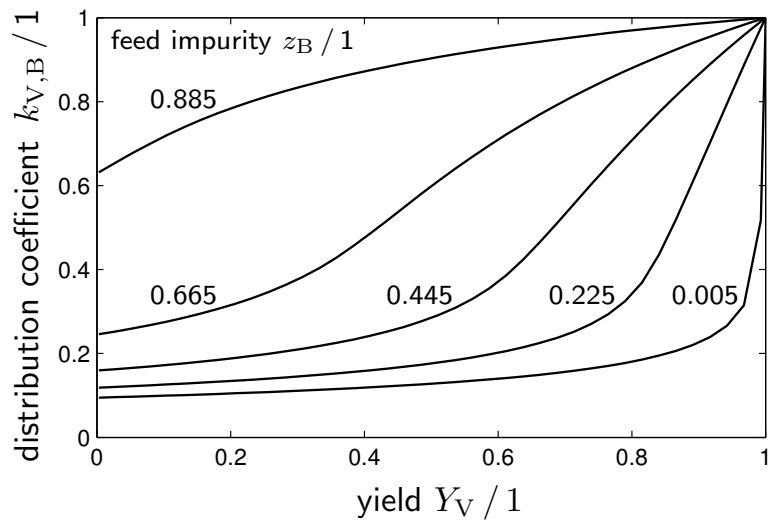

Figure 18: Evaporative crystallization. Intermediate product removal: tapered structure with 50 stages. Distribution of impurity to product depending on yield for different feed impurities. Lower distribution coefficient for lower feed impurity.

\subsection{General design guidelines}

All considered processes offer substantial reductions of separation effort when using multistage configurations different from countercurrent cascades. Improved configurations are found at boundaries of parameter regions where optimal countercurrent cascades have different feed positions. The largest reductions of separation effort are observed where optimal countercurrent cascades have two stages. Configuration (a) in Figure 14 rearranged for better readability in Figure 19, is globally optimal in terms of separation effort for all considered processes. Insight to economical design of multistage separation processes is gained as well, since the separation effort is an indicator for the economical viability of a process. Recall that reduced separation effort gives a measure for potential reduction of operating and investment cost.

Based on the findings for different processes, following design guidelines for general multistage separation processes using networks of units with the same separation characteristic are proposed. 
If parameter studies for optimal countercurrent cascades are available, the search for improved configurations should be restricted to regions of interest, i. e. boundaries of parameter regions with different optimal countercurrent feed positions and a small number of stages. Optimization of countercurrent cascades has a significantly lower computational cost than optimization of the general superstructure.

If parameter studies for optimal countercurrent cascades are not available, the set of candidate configurations should be extended to include the three-stage configuration depicted in Figure 19 The conditions for improved configurations using tapered structures should be checked, e.g. analytically or via optimization using the process model. If the conditions are fulfilled, the set of candidate configurations should be extended to include configurations generated by replacing one or more single stages of candidate solutions with tapered structures. This step can be omitted if tapered configurations are regarded anyway in the actual implementation of the considered process to account for technical limitations of single units.

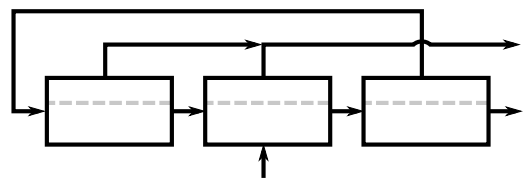

Figure 19: Common optimal alternative configuration for all considered processes.

\section{Conclusion}

Multistage configurations for three separation processes, namely cooling crystallization, evaporative crystallization and filtration, are studied using global optimization. Design guidelines for multistage separation processes are derived from analyzing the influence of process parameters on globally optimal configurations for all processes. The findings are qualitatively equivalent in all three cases, allowing the generalization of corresponding design guidelines. For considerable parts of the parameter domain, single stages or countercurrent cascades 
are the favored configurations. In large parameter regions two types of configurations are identified that substantially reduce the separation effort compared to countercurrent cascades. Design steps with more detailed models need to follow after the conceptual design considered in this work. However, the detailed design should account for additional process candidates identified with our systematic approach. Subsequent design steps should also account for possible performance limiting effects of real separation units, e.g. by considering each separation stage to comprise multiple separation modules in a tapered structure.

\section{Acknowledgements}

This work is part of the Collaborative Research Center "Integrated Chemical Processes in Liquid Multiphase Systems". Financial support by the Deutsche Forschungsgemeinschaft (DFG) is gratefully acknowledged through TRR 63.

\section{References}

[1] W. L. McCabe, J. C. Smith, P. Harriott, Unit Operations of Chemical Engineering, 5th Edition, McGraw-Hill, London, 1993.

[2] G. Wellinghoff, K. Wintermantel, Melt crystallization - theoretical presumptions and technical limitations [Schmelzkristallisation - theoretische Voraussetzungen und technische Grenzen], Chemie-Ingenieur-Technik 63 (9) (1991) 881-888, 89. doi:10.1002/cite.330630903.

[3] S. W. Gilbert, Melt crystallization: Process analysis and optimization, AIChE Journal 37 (8) (1991) 1205-1218. doi:10.1002/aic.690370810

[4] C. Wallert, W. Marquardt, J. T. Leu, J. Strube, Design and optimization of layer crystallization processes, in: L. Puigjaner, A. Espuña (Eds.), European Symposium on Computer-Aided Process Engineering-15, 38th European Symposium of the Working Party on Computer Aided Process Engineering, Vol. 20 of Computer Aided Chemical Engineering, Elsevier, 2005, pp. 871-876. doi:10.1016/S1570-7946(05)80267-6. 
[5] J. Micovic, T. Beierling, P. Lutze, G. Sadowski, A. Górak, Design of hybrid distillation/melt crystallisation processes for separation of close boiling mixtures, Chemical Engineering and Processing: Process Intensification 67 (2013) 16-24. doi:10.1016/j.cep.2012.07.012.

[6] E. Temmel, U. Müller, D. Grawe, R. Eilers, H. Lorenz, A. SeidelMorgenstern, Equilibrium model of a continuous crystallization process for separation of substances exhibiting solid solutions, Chemical Engineering \& Technology 35 (6) (2012) 980-985. doi:10.1002/ceat.201200002.

[7] E. Temmel, S. Wloch, U. Müller, D. Grawe, R. Eilers, H. Lorenz, A. SeidelMorgenstern, Aufreinigung durch mehrstufige Gegenstromkristallisation zur Herstellung hochreiner Pharmazeutika, Chemie Ingenieur Technik 85 (10) (2013) 1581-1588. doi:10.1002/cite.201200244.

[8] S. Münzberg, H. Lorenz, A. Seidel-Morgenstern, Multistage countercurrent crystallization for the separation of solid solutions, Chemical Engineering \& Technology 39 (7) (2016) 1242-1250. doi:10.1002/ceat.201600093.

[9] M. Olbrycht, M. Balawejder, K. Matuła, W. Piatkowski, D. Antos, Multistage cross-current and countercurrent flow crystallization for separation of racemic 2-methylbutanoic acid, Industrial \& Engineering Chemistry Research 53 (41) (2014) 15990-15999. doi:10.1021/ie501703w.

[10] J. C.-T. Lin, A. G. Livingston, Nanofiltration membrane cascade for continuous solvent exchange, Chemical Engineering Science 62 (10) (2007) 2728-2736. doi:10.1016/j.ces.2006.08.004.

[11] A. Caus, L. Braeken, K. Boussu, B. Van der Bruggen, The use of integrated 425 countercurrent nanofiltration cascades for advanced separations, Journal

1. of Chemical Technology \& Biotechnology 84 (3) (2009) 391-398. doi: $10.1002 /$ jctb. 2052 .

[12] K. Tanaka, K. Matsui, T. Hori, H. Iwahashi, K. Takeuchi, Y. Ito, World's 
first large triple-pass RO seawater desalination plant., Technical Review 46, Mitsubishi Heavy Industries (2009).

[13] E. Gasia-Bruch, M. Busch, V. García-Molina, U. Kolbe, Dual membrane systems in seawater desalination: Drivers for selection and field experiences, 2011, presented in IDA Desalination Industry Action for Good, Portofino, 2011.

[14] K. McMordie Stoughton, X. Duan, E. M. Wendel, Reverse osmosis optimization, Tech. rep., Pacific Northwest National Laboratory, Richland, Washington 99352 (8 2013). doi:10.2172/1095449.

[15] D. Cingolani, F. Fatone, N. Frison, M. Spinelli, A. Eusebi, Pilot-scale multistage reverse osmosis (DT-RO) for water recovery from landfill leachate, Waste Management 76 (2018) 566-574. doi:10.1016/j.wasman.2018.03. 014.

[16] R. Khalilpour, K. Mumford, H. Zhai, A. Abbas, G. Stevens, E. S. Rubin, Membrane-based carbon capture from flue gas: a review, Journal of Cleaner Production 103 (2015) 286-300. doi:10.1016/j.jclepro.2014.10.050.

[17] B. Bhide, S. Stern, Membrane processes for the removal of acid gases from natural gas. I. process configurations and optimization of operating conditions, Journal of Membrane Science 81 (3) (1993) 209-237. doi:10.1016/0376-7388(93)85175-V.

[18] A. K. Datta, P. K. Sen, Optimization of membrane unit for removing carbon dioxide from natural gas, Journal of Membrane Science 283 (1) (2006) 291300. doi:10.1016/j.memsci.2006.06.043.

[19] P. Gabrielli, M. Gazzani, M. Mazzotti, On the optimal design of membranebased gas separation processes, Journal of Membrane Science 526 (Supplement C) (2017) 118-130. doi:10.1016/j.memsci.2016.11.022. 
$[22]$

口

[23] M. Scholz, Membrane based biogas upgrading processes, Ph.D. thesis, Rheinisch-Westfälische Technische Hochschule Aachen (2013).

[24] M. Scholz, M. Alders, T. Lohaus, M. Wessling, Structural optimization of membrane-based biogas upgrading processes, Journal of Membrane Science 474 (Supplement C) (2015) 1-10. doi:10.1016/j.memsci.2014.08.032.

[25] C. Kunde, A. Kienle, Deterministic global optimization of multistage melt crystallization processes in hydroformylation, Computer Aided Chemical

【 Engineering 37 (2015) 1061-1066. doi:10.1016/B978-0-444-63577-8. $50022-\mathrm{X}$.

475

[26] C. Kunde, D. Michaels, J. Micovic, P. Lutze, A. Górak, A. Kienle, Deterministic global optimization in conceptual process design of distillation and melt crystallization, Chemical Engineering and Processing: Process Intensification 99 (2016) 132-142. doi:10.1016/j.cep.2015.09.010.

[27] M. M. El-Halwagi, V. Manousiouthakis, Synthesis of mass exchange net480 works, AIChE Journal 35 (8) (1989) 1233-1244. doi:10.1002/aic. 690350802 . 
[28] I. E. Grossmann, J. A. Caballero, H. Yeomans, Mathematical programming approaches to the synthesis of chemical process systems, Korean Journal of Chemical Engineering 16 (4) (1999) 407-426. doi:10.1007/BF02698263.

[29] Z. Chen, J. Wang, Heat, mass, and work exchange networks, Frontiers of Chemical Science and Engineering 6 (4) (2012) 484. doi:10.1007/ s11705-012-1221-5.

[30] A. Lejeune, M. Rabiller-Baudry, T. Renouard, B. Balannec, Y. Liu, J. Augello, D. Wolbert, Assessment and potential of membrane cascades for organic solvent nanofiltration of hydroformylation media through a graphical representation composed of performance maps, Chemical Engineering Science 183 (2018) 240-259. doi:10.1016/j.ces.2018.03.025.

[31] M. G. Marcovecchio, P. A. Aguirre, N. J. Scenna, Global optimal design of reverse osmosis networks for seawater desalination: modeling and algorithm, Desalination 184 (1) (2005) 259-271. doi:10.1016/j.desal.2005. 03.056

[32] F. Evangelista, Design and performance of two-stage reverse osmosis plant, Chemical Engineering and Processing: Process Intensification 25 (3) (1989) 119-125. doi:10.1016/0255-2701(89)80001-7.

[33] A. Hafez, S. El-Mariharawy, Design and performance of the two-stage/twopass RO membrane system for chromium removal from tannery wastewater. Part 3, Desalination 165 (2004) 141-151. doi:10.1016/j.desal.2004.06. 016.

[34] A. Zhu, P. D. Christofides, Y. Cohen, Minimization of energy consumption for a two-pass membrane desalination: Effect of energy recovery, membrane rejection and retentate recycling, Journal of Membrane Science 339 (1) (2009) 126-137. doi:10.1016/j.memsci.2009.04.039.

[35] N. Hilal, G. Kim, C. Somerfield, Boron removal from saline water: A com- 
prehensive review, Desalination 273 (1) (2011) 23-35. doi:10.1016/j. desal.2010.05.012.

[36] N. Voros, Z. Maroulis, D. Marinos-Kouris, Optimization of reverse osmosis networks for seawater desalination, Computers \& Chemical Engineering 20 (1996) 345-350. doi:10.1016/0098-1354(96)00068-3.

[37] J. E. Blesing, C. Pelekani, Seawater desalination: Sustainable solution to 515 world water shortage, Water e-Journal 1 (1) (2016) 1-8. doi:10.21139/ wej.2016.003

[38] R. C. Cheng, T. J. Tseng, K. L. Wattier, Two-pass nanofiltration seawater desalination prototype testing and evaluation, Desalination and Water Purification Research and Development Report 158, Long Beach Water Department, 1800 E. Wardlow Ave. Long Beach, CA 90807 (2013).

[39] C. Kunde, Global optimization in conceptual process design, Ph.D. thesis, Otto-von-Guericke University Magdeburg (2017).

[40] A. Mersmann (Ed.), Crystallization technology handbook, 2nd Edition, Marcel Dekker, Inc., 2001.

[41] J. Ulrich, J. Bierwirth, S. Henning, Solid layer melt crystallization, 1. Separation \& Purification Reviews 25 (1) (1996) 1-45. doi:10.1080/ 03602549608006625 .

[42] J. Wijmans, R. Baker, The solution-diffusion model: a review, Journal of Membrane Science 107 (1995) 1-21.

${ }_{530}$ [43] M. Tawarmalani, N. Sahinidis, A polyhedral branch-and-cut approach to 1 global optimization, Mathematical Programming 103 (2005) 225-249. doi: 10.1007/s10107-005-0581-8.

[44] J. Micovic, K. Werth, P. Lutze, Hybrid separations combining distillation and organic solvent nanofiltration for separation of wide boiling mixtures, 


\section{Appendix A. Model for organophilic nanofiltration}

Membrane unit. The specific permeate flow $v_{i}$ across the membrane according to the solution-diffusion model [42] is

$$
v_{i}=k_{\mathrm{P}, i}\left(x_{i}-y_{i} \exp \left(-\frac{\nu_{i} \Delta P}{\mathrm{R} T}\right)\right)
$$

with the molar fraction of component $i$ on the feed side $x_{i}$ and on the permeate side $y_{i}$, pressure difference $\Delta P$ and temperature $T$. The parameter $k_{\mathrm{P}, i}$ specifies the permeability of the membrane with regard to component $i$. It is dependent on the considered component, membrane type, membrane geometry and temperature. The molar volume of a component is denoted as $\nu_{i}$. The retentate is collected on the feed side at the end of the unit and the permeate over the whole length of the membrane. The overall permeate flow for a filtration unit is calculated by integrating the specific permeate flow $v_{i}$ over the membrane area $A$ in flow direction $Z$.

$$
V_{i}=\frac{\partial A}{\partial Z} \int_{Z} v_{i} \mathrm{~d} Z
$$

The integral is solved by using $K+1$ equally spaced discretization points $Z_{k}, k=$ $0, \ldots, K$, as

$$
V_{i}=\sum_{k=0}^{K-1} \frac{\partial A}{\partial Z} \int_{Z_{k}}^{Z_{k+1}} v_{i} \mathrm{~d} Z
$$

and approximated by assuming a linear profile for $v_{i}$ between discretization points:

$$
\int_{Z_{k}}^{Z_{k+1}} v_{i} \mathrm{~d} Z \approx\left(\left.0.5 v_{i}\right|^{k}+\left.0.5 v_{i}\right|^{k+1}\right) \Delta Z .
$$


With $\Delta A=\frac{\partial A}{\partial Z} \Delta Z$, the integral permeate flow for each membrane unit $n=$ $1, \ldots, N$ separating a feed molar flow $F_{i, n}$ into a permeate molar flow $V_{i, n}$ and a retentate molar flow $L_{i, n}$ for components $i=\mathrm{A}, \mathrm{B}$ as in Figure 5 is then calculated as

$$
\begin{aligned}
V_{i, n} & =\left.\sum_{k=0}^{K-1} V_{i, n}\right|^{k}=\sum_{k=0}^{K-1}\left(\left.0.5 v_{i, n}\right|^{k}+\left.0.5 v_{i, n}\right|^{k+1}\right) \Delta A_{n}, \\
\left.v_{i, n}\right|^{k} & =k_{\mathrm{P}, i}\left(\left.x_{i, n}\right|^{k}-\left.y_{i, n}\right|^{k} \exp \left(-\frac{\nu_{i} \Delta P}{\mathrm{R} T}\right)\right),
\end{aligned}
$$

with molar fractions of component $i=\mathrm{A}, \mathrm{B}$ on the feed side $x_{i}, z_{i}$ and on the permeate side $y_{i}$ defined by

$$
\begin{aligned}
\left.\left.x_{i, n}\right|^{k} \sum_{j=\mathrm{A}, \mathrm{B}} L_{j, n}\right|^{k} & =\left.L_{i, n}\right|^{k}, \\
\left.\left.y_{i, n}\right|^{k} \sum_{j=\mathrm{A}, \mathrm{B}} v_{j, n}\right|^{k} & =\left.v_{i, n}\right|^{k} .
\end{aligned}
$$

Mass balances for the discretization volumes give

$$
\left.L_{i, n}\right|^{k}=\left.L_{i, n}\right|^{k-1}-\left.V_{i, n}\right|^{k}
$$

and boundary conditions

$$
\begin{aligned}
& L_{i, n}=\left.L_{i, n}\right|^{K}, \\
& F_{i, n}=\left.L_{i, n}\right|^{0} .
\end{aligned}
$$

The total membrane area is

$$
A_{n}=K \Delta A_{n} .
$$

Compatibility with the definition of general separation units requires

$$
\begin{gathered}
F_{n}=F_{\mathrm{A}, n}+F_{\mathrm{B}, n}, \\
V_{n}=V_{\mathrm{A}, n}+V_{\mathrm{B}, n}, \\
L_{n}=L_{\mathrm{A}, n}+L_{\mathrm{B}, n} .
\end{gathered}
$$

The yield $Y_{\mathrm{V}}$ and the distribution coefficient $k_{\mathrm{V}, \mathrm{B}}$ are calculated from the definition of the general separation unit. 
Appendix B. Model for evaporative crystallization

Crystallization unit. The model for the crystallization unit from [8] adapted for steady-state operation is written in terms of the primary component $\mathrm{A}$, the solvent $\mathrm{S}$ and all components without solvent $\mathrm{AB}$, with according index $i=\mathrm{A}, \mathrm{AB}, \mathrm{S}$. Quantities at the end of step 1, i. e. mixing of feed flows, for the process illustrated in Figure 7 are calculated from the unit feed as

$$
\begin{aligned}
L_{\mathrm{AB}, n}^{(1)} & =F_{n}, \\
L_{\mathrm{A}, n}^{(1)} & =F_{\mathrm{A}, n},
\end{aligned}
$$

with the total mass flow without solvent in the liquid phase $L_{\mathrm{AB}, n}^{(1)}$ and the mass flow of the primary component $L_{\mathrm{A}, n}^{(1)}$.

The required amount of solvent $L_{\mathrm{S}, n}^{(2)}$ for step 2, i. e. dissolving all solid in the feed, is determined via the phase equilibrium. The phase equilibrium is given in [8] by polynomial fits to experimental data using mass fractions in the feed mass flow $x_{i, n}$ and in the virtual solid phase $y_{i, n}$. The flows for non-solvent components are the same between step 1 and step 2 , so $L_{\mathrm{j}, n}^{(1)}=L_{\mathrm{j}, n}^{(2)}$ for $j=\mathrm{A}, \mathrm{AB}$. Resulting equations for the dissolution step are given below.

$$
\begin{aligned}
L_{\mathrm{A}, n}^{(2)} & =x_{\mathrm{A}, n}^{(2)}\left(L_{\mathrm{AB}, n}^{(2)}+L_{\mathrm{S}, n}^{(2)}\right) \\
L_{\mathrm{S}, n}^{(2)} & =x_{\mathrm{S}, n}^{(2)}\left(L_{\mathrm{AB}, n}^{(2)}+L_{\mathrm{S}, n}^{(2)}\right) \\
x_{\mathrm{A}, n}^{(2)} & =\sum_{k=0}^{3} a_{\mathrm{A}, k}\left(y_{\mathrm{A}, n}^{(2)}\right)^{k} \\
x_{\mathrm{S}, n}^{(2)} & =\sum_{k=0}^{3} a_{\mathrm{S}, k}\left(y_{\mathrm{A}, n}^{(2)}\right)^{k}
\end{aligned}
$$

Solvent is removed to induce crystallization in step 3. Crystallization proceeds until the solution is in equilibrium with the solid phase $V$, i.e. the crystals. Solvent content after the crystallization step is determined in the same way as 
for the dissolution step.

$$
\begin{aligned}
L_{\mathrm{A}, n}^{(3)} & =x_{\mathrm{A}, n}^{(3)}\left(L_{\mathrm{AB}, n}^{(3)}+L_{\mathrm{S}, n}^{(3)}\right) \\
L_{\mathrm{S}, n}^{(3)} & =x_{\mathrm{S}, n}^{(3)}\left(L_{\mathrm{AB}, n}^{(3)}+L_{\mathrm{S}, n}^{(3)}\right) \\
x_{\mathrm{A}, n}^{(3)} & =\sum_{k=0}^{3} a_{\mathrm{A}, k}\left(y_{\mathrm{A}, n}^{(3)}\right)^{k} \\
x_{\mathrm{S}, n}^{(3)} & =\sum_{k=0}^{3} a_{\mathrm{S}, k}\left(y_{\mathrm{A}, n}^{(3)}\right)^{k} \\
V_{\mathrm{A}, n}^{(3)} & =y_{\mathrm{A}, n}^{(3)} V_{\mathrm{AB}, n}^{(3)}
\end{aligned}
$$

The remaining liquid is drained from the unit in step 4. However, some liquid may adhere to the crystals. With zero solvent content in the crystals themselves $V_{\mathrm{S}, n}^{(3)}=0$, the crystal product including adherent liquid proportional to the crystal mass $V_{\mathrm{AB}, n}^{(3)}$ is calculated as follows using ratio parameter $\kappa$. Note that the composition of the liquid phase does not change in this step.

$$
\begin{aligned}
V_{i, n}^{(4)} & =V_{i, n}^{(3)}+\kappa V_{\mathrm{AB}, n}^{(3)} x_{i, n}^{(3)} \\
L_{i, n}^{(4)} & =L_{i, n}^{(3)}-\kappa V_{\mathrm{AB}, n}^{(3)} x_{i, n}^{(3)} \\
x_{i, n}^{(4)} & =x_{i, n}^{(3)}
\end{aligned}
$$

Main product $V_{i, n}$ and secondary product $L_{i, n}$ of the unit are the corresponding products of step 4 , i. e.

$$
\begin{aligned}
& V_{i, n}=V_{i, n}^{(4)}, \\
& L_{i, n}=L_{i, n}^{(4)} .
\end{aligned}
$$

The evaporated solvent for each stage is determined by

$$
S_{n}=\max \left(L_{\mathrm{S}, n}^{(1)}, L_{\mathrm{S}, n}^{(2)}\right)-L_{\mathrm{S}, n}^{(3)} .
$$

Compatibility with the definition of general separation units requires

$$
\begin{gathered}
F_{n}=F_{\mathrm{A}, n}+F_{\mathrm{B}, n}, \\
V_{n}=V_{\mathrm{A}, n}+V_{\mathrm{B}, n}, \\
L_{n}=L_{\mathrm{A}, n}+L_{\mathrm{B}, n} .
\end{gathered}
$$


The yield $Y_{\mathrm{V}}$ and the distribution coefficient $k_{\mathrm{V}, \mathrm{B}}$ are calculated from the definition of the general separation unit.

Selectivity scaling. At equilibrium, the composition in the liquid phase is a function of the composition in the solid phase $x_{\mathrm{A}}=x_{\mathrm{A}}\left(y_{\mathrm{B}}\right), x_{\mathrm{B}}=x_{\mathrm{B}}\left(y_{\mathrm{B}}\right)$. Subscript "scaled" describes values after selectivity scaling.

Some intermediate steps are omitted, see [39] for more details.

The separation efficiency is described by selectivity $\alpha$ in analogy to the relative volatility in distillation, see [8]. The unscaled selectivity of component B is

$$
\alpha_{\mathrm{B}}=\frac{y_{\mathrm{B}} x_{\mathrm{A}}}{y_{\mathrm{A}} x_{\mathrm{B}}},
$$

with $\alpha_{\mathrm{B}} \in[0,1]$, and values of $\alpha_{\mathrm{B}}$ close to one for low separation efficiency and values close to zero for high separation efficiency. Note that this equation is not defined for $y=0$ for any component.

The selectivity scaling is designed to range between no separation and complete separation, including the original model as an intermediate case. Furthermore, the solubility line is preserved for all scaling values. This is achieved by calculating a new value for the selectivity $\alpha_{\mathrm{B}}$ as

$$
\alpha_{\mathrm{B}, \mathrm{scaled}}=\frac{k_{\alpha} \alpha_{\mathrm{B}}}{\left(1-\alpha_{\mathrm{B}}\right)+k_{\alpha} \alpha_{\mathrm{B}}},
$$

with $k_{\alpha} \in[0, \infty)$ and

$$
\begin{aligned}
& \alpha_{\mathrm{B}, \text { scaled }}\left(k_{\alpha}=0\right)=0, \\
& \alpha_{\mathrm{B}, \text { scaled }}\left(k_{\alpha}=1\right)=\alpha_{\mathrm{B}}, \\
& \alpha_{\mathrm{B}, \text { scaled }}\left(k_{\alpha} \rightarrow \infty\right)=1 .
\end{aligned}
$$

This intermediate result is used to calculate a new value of the solid composition $y_{\mathrm{B}, \mathrm{scaled}}$ as

$$
y_{\mathrm{B}, \mathrm{scaled}}=\frac{\alpha_{\mathrm{B}, \text { scaled }} x_{\mathrm{B}}}{x_{\mathrm{A}}+\alpha_{\mathrm{B}, \mathrm{scaled}} x_{\mathrm{B}}} .
$$

As a single equation, the scaled solid composition $y_{\mathrm{B}, \text { scaled }}$ is

$$
y_{\mathrm{B}, \text { scaled }}=\frac{k_{\alpha} y_{\mathrm{B}} x_{\mathrm{B}}}{\left(k_{\alpha}-1\right) y_{\mathrm{B}}\left(x_{\mathrm{A}}+x_{\mathrm{B}}\right)+x_{\mathrm{B}}} .
$$


Substitution of polynomials $x_{\mathrm{B}}\left(y_{\mathrm{B}}\right)$ and $x_{\mathrm{A}}\left(y_{\mathrm{B}}\right)$ leads to a univariate function with polynomials in the numerator and the denominator.

$$
y_{\mathrm{B}, \text { scaled }}=\frac{k_{\alpha} x_{\mathrm{B}}}{\left(k_{\alpha}-1\right)\left(x_{\mathrm{A}}+x_{\mathrm{B}}\right)+\frac{x_{\mathrm{B}}}{y_{\mathrm{B}}}}
$$

\section{Appendix C. Correlations for general separation units}

Main product yield

$$
V=Y_{\mathrm{V}} F \text {. }
$$

Distribution of secondary component to main product

$$
y_{\mathrm{B}}=k_{\mathrm{V}, \mathrm{B}} z_{\mathrm{B}}
$$

Mass balances

$$
F=V+L, \quad F_{\mathrm{B}}=V_{\mathrm{B}}+L_{\mathrm{B}}, \quad F_{\mathrm{A}}=V_{\mathrm{A}}+L_{\mathrm{A}} .
$$

Summation conditions

$$
z_{\mathrm{B}}+z_{\mathrm{A}}=1, \quad x_{\mathrm{B}}+x_{\mathrm{A}}=1, \quad y_{\mathrm{B}}+y_{\mathrm{A}}=1 .
$$

Recovery of secondary component in main product

$$
V_{\mathrm{B}}=k_{\mathrm{V}, \mathrm{B}} Y_{\mathrm{V}} F_{\mathrm{B}} .
$$

Recovery of main component in main product

$$
V_{\mathrm{A}}=\frac{\left(1-k_{\mathrm{V}, \mathrm{B}} z_{\mathrm{B}}\right) Y_{\mathrm{V}}}{1-z_{\mathrm{B}}} F_{\mathrm{A}} .
$$

Distribution of main component to main product

$$
y_{\mathrm{A}}=\left(1-k_{\mathrm{V}, \mathrm{B}} z_{\mathrm{B}}\right) .
$$

Secondary product yield

$$
L=Y_{\mathrm{L}} F, \quad Y_{\mathrm{L}}=1-Y_{\mathrm{V}} .
$$


Recovery of secondary component in secondary product

$$
L_{\mathrm{B}}=\left(1-k_{\mathrm{V}, \mathrm{B}} Y_{\mathrm{V}}\right) F_{\mathrm{B}} .
$$

Distribution of secondary component to secondary product

$$
x_{\mathrm{B}}=k_{\mathrm{L}, \mathrm{B}} z_{\mathrm{B}}, \quad k_{\mathrm{L}, \mathrm{B}}=\frac{1-k_{\mathrm{V}, \mathrm{B}} Y_{\mathrm{V}}}{1-Y_{\mathrm{V}}} .
$$


Nomenclature

variable description, unit

General

$i, j \quad$ component index, 1

$l, n \quad$ stage number index, 1

$J$ objective function, 1

$k$ distribution coefficient, 1

$N$ number of stages, 1

$N_{\mathrm{x}} \quad$ number of continuous variables, 1

$N_{\mathrm{y}} \quad$ number of binary variables, 1

$Y$ yield, 1

$F, V, L$ molar flow (mass flow), $\mathrm{mol} \mathrm{s}^{-1}\left(\mathrm{~kg} \mathrm{~s}^{-1}\right)$

$z, y, x$ molar fraction (mass fraction), 1

$\bar{F}, \bar{V}, \bar{L}$ overall molar flow (mass flow), $\mathrm{mol} \mathrm{s}^{-1}\left(\mathrm{~kg} \mathrm{~s}^{-1}\right)$

$\bar{z}, \bar{y}, \bar{x} \quad$ overall molar fraction (mass fraction), 1

$\bar{z}_{\text {norm }}$ normalized feed composition, 1

$\beta$ binary connection variable, 1

Cooling crystallization

$k_{\text {diff }}$ differential distribution coefficient, 1

Filtration

$A$ area, $\mathrm{m}^{2}$

$k_{\mathrm{P}} \quad$ permeability, $\mathrm{mol} \mathrm{m}^{-2} \mathrm{~s}^{-1}$

$k_{\mathrm{P}, \mathrm{B}, \text { norm }} \quad$ normalized permeability of $\mathrm{B}, 1$

$k_{\mathrm{P}, \mathrm{B}, \mathrm{ref}} \quad$ reference permeability of $\mathrm{B}, \mathrm{mol} \mathrm{m}^{-2} \mathrm{~s}^{-1}$

$K$ number of discretization elements, 1

$\mathrm{R}$ gas constant, $8.314 \mathrm{~J} \mathrm{~mol}^{-1} \mathrm{~K}^{-1}$

$v$ specific permeate flow, $\mathrm{mol} \mathrm{m}^{-2} \mathrm{~s}^{-1}$

$T$ temperature, $\mathrm{K}$

$\Delta P$ pressure difference, $\mathrm{Pa}$

$\nu$ molar volume, $\mathrm{m}^{-3} \mathrm{~mol}^{-1}$

Evaporative crystallization 4

a phase equilibrium parameter, 1

$k_{\alpha} \quad$ scaling parameter, 1

$S$ evaporated solvent mass flow, $\mathrm{kg} \mathrm{s}^{-1}$

$\kappa$ fraction of adhering liquid, 1 
Table 1: Cooling crystallization. Parameter and domain specifications.

\begin{aligned} & parameter value \\ & \hline$k_{\text {diff }}\{0.01,0.02, \ldots, 0.5\} \\ & \bar{z}_{\mathrm{B}}\{0.06,0.08, \ldots, 0.84\} \\ & N\{1,2,3,4\} \\ & \bar{F} 1 \mathrm{~mol} \mathrm{~s}^{-1} \\ & x_{\mathrm{B}}^{\max } 0.9 \\ &$\hline variable domain \\ & \hline $\bar{y}_{\mathrm{B}} {[0,0.05] } \\ & \bar{x}_{\mathrm{B}} {[0.85,1] } \\ & Y {[0,1] } \\ & V, L, F {\left[0 \mathrm{~mol} \mathrm{~s} \mathrm{~s}^{-1}, 20 \mathrm{~mol} \mathrm{~s}^{-1}\right] } \\ & \beta\{0,1\} \\ &$\hline\end{aligned}


Table 2: Filtration. Parameter and domain specifications.

\begin{tabular}{rl}
\hline parameter & value \\
\hline$k_{\mathrm{P}, \mathrm{B}, \text { norm }}$ & $\{0.05,0.1, \ldots, 2\}$ \\
$\bar{z}_{\text {norm }}$ & $\{0.01,0.015, \ldots, 0.095\}$ \\
$N$ & $\{1,2,3\}$ \\
$\Delta P$ & $35 \cdot 10^{5} \mathrm{~Pa}$ \\
$T$ & $313.15 \mathrm{~K}$ \\
$\bar{F}$ & $1 \mathrm{~mol} \mathrm{~s}^{-1}$ \\
$k_{\mathrm{P}, \mathrm{A}}$ & $0.5411 \mathrm{~mol} \mathrm{~s}^{-1} \mathrm{~m}^{-2}{ }^{*}$ \\
$k_{\mathrm{P}, \mathrm{B}, \text { ref }}$ & $0.02104 \mathrm{~mol} \mathrm{~s}^{-1} \mathrm{~m}^{-2}{ }^{*}$ \\
$\nu_{\mathrm{A}}$ & $1.95446 \cdot 10^{-4} \mathrm{~m}^{3} \mathrm{~mol}^{-1}$ \\
$\nu_{\mathrm{B}}$ & $4.69981 \cdot 10^{-4} \mathrm{~m}^{3} \mathrm{~mol}^{-1}$ \\
$K$ & 2 \\
\hline variable & domain \\
\hline $\bar{y}_{\mathrm{B}}$ & {$[0,0.005]$} \\
$\bar{x}_{\mathrm{B}}$ & {$[0.1,1]$} \\
$y, x, z$ & {$[0,1]$} \\
$V, L, F$ & {$\left[0 \mathrm{~mol} \mathrm{~s}^{-1}, 10 \mathrm{~mol} \mathrm{~s}^{-1}\right]$} \\
$\beta$ & $\{0,1\}$ \\
$v_{\mathrm{i}}$ & {$\left[0 \mathrm{~mol} \mathrm{~s}^{-1} \mathrm{~m}^{-2}, k_{\mathrm{P}, i}\right]$} \\
$A$ & {$\left[0 \mathrm{~m}^{2}, 100 \mathrm{~m}^{2}\right]$} \\
\hline${ }^{*}[44]$ \\
\hline
\end{tabular}


Table 3: Evaporative crystallization. Parameter and domain specifications.

\begin{tabular}{rl} 
parameter & value \\
\hline$\left(a_{\mathrm{A}, 0}, a_{\mathrm{A}, 1}\right)$ & $(0,0.1949)^{*}$ \\
$\left(a_{\mathrm{A}, 2}, a_{\mathrm{A}, 3}\right)$ & $(-0.2905,0.2474)^{*}$ \\
$\left(a_{\mathrm{S}, 0}, a_{\mathrm{S}, 1}\right)$ & $(0.5149,0.3131)^{*}$ \\
$\left(a_{\mathrm{S}, 2}, a_{\mathrm{S}, 3}\right)$ & $(-0.9066,0.9268)^{*}$ \\
$k_{\alpha}$ & $\{0.1,0.2, \ldots, 2\}$ \\
$\bar{z}_{\text {norm }}$ & $\{0.0833,0.1667, \ldots, 0.9167\}$ \\
$N$ & $\{1,2,3\}$ \\
$\bar{F}$ & $1 \mathrm{~kg} \mathrm{~s}^{-1}$ \\
$\kappa$ & 0.01 \\
$\bar{y}_{\mathrm{B}}$ & {$[0,0.1]$} \\
$\bar{z}_{\mathrm{B}}$ & {$[0.7,1]$} \\
$\bar{F}_{\mathrm{S}}$ & $0 \mathrm{~kg} \mathrm{~s}^{-1}$ \\
\hline variable & domain \\
\hline$y, z$ & {$[0,1]$} \\
$\beta$ & $\{0,1\}$ \\
$V, L, F, S$ & {$\left[0 \mathrm{~kg} \mathrm{~s}^{-1}, 20 \mathrm{~kg} \mathrm{~s}^{-1}\right]$} \\
$x_{\mathrm{A}}$ & {$[0,0.1518]$} \\
$x_{\mathrm{S}}$ & {$[0.5149,0.8482]$} \\
\hline & ${ }^{*}[8$
\end{tabular}

${ }^{*}$ [8] 